Iranian Journal of Pathology | ISSN: 2345-3656

\title{
Is There A Correlation Between COVID-19 and Hepatitis A and Hepatitis E Serum Antibody Level?
}

\author{
Alireza Abdollahi1 $^{1 D}$, Samaneh Salarvand ${ }^{1 *}$ iD, Vahid Mehrtash1 ${ }^{1 D}$, Bita Jafarzadeh1 ${ }^{\text {iD }}$, \\ Reza Ghalehtaki ${ }^{2}$, Saeed Nateghi ${ }^{3}$
}

1. Department of Pathology, Imam Khomeini Hospital Complex, Tehran University of Medical Sciences, Tehran, Iran

2. Radiation Oncology Research Center, Cancer Institute, Tehran University of Medical Sciences, Tehran, Iran

3. Department of Cardiology, Tehran University of Medical Sciences, Tehran, Iran

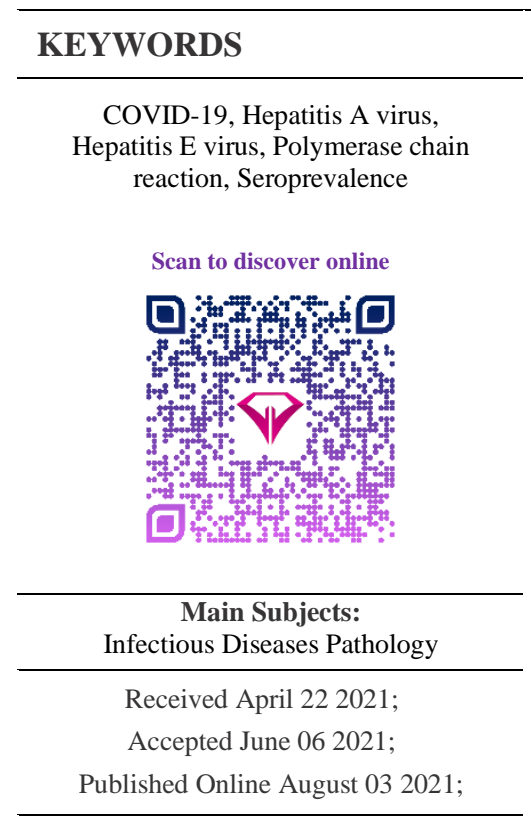

10.30699/IJP.2021.528077.2615

\section{ABSTRACT}

Background \& Objective: The prevalence of COVID-19 and its severity have been observed to be on a lower level in underdeveloped countries with poorer standards of hygiene. This disparity may be attributed to the higher seroprevalence of other viral diseases, which can result in the presence of antibodies protective against COVID-19. Two of the widespread diseases in such countries are infection to hepatitis $\mathrm{A}$ and $\mathrm{E}$ viruses (HAV and HEV). In the present study, we explored the relationship between the level of antibodies against these viruses and the susceptibility to COVID-19.

\begin{abstract}
Methods: Ninety patients were studied in two groups of controls and cases, each consisting 45 individuals. The cases were patients with the clinical symptoms of COVID19 and positive RT-PCR test results. The controls were individuals referred to the respiratory triage of Imam Khomeini Hospital Complex and were not demonstrating relevant clinical symptoms of COVID-19 and their RT-PCR test results were negative. Levels of HAV and HEV antibodies were measured and compared in these two groups.
\end{abstract}

Results: The median of HAV antibody level was 13.6 (IQR=11.5-16.9) and 13.2 (IQR $=10.7-14.7)$ in cases and controls, respectively, showing no statistically significant difference $(P=0.1)$. Likewise, the median of HEV antibody level was 6.7 (IQR=5.37.1) and 7.1 (IQR=6.3-7.5) in cases and controls, respectively, which again showed no statistically significant difference $(P=0.41)$.

Conclusion: The present study was carried out in a region with a relatively high prevalence of HAV and HEV infections. Contrary to our expectations, no statistically significant relationship was observed between the levels of antibodies against these viruses and the susceptibility to COVID-19. Further studies with larger sample sizes and in other countries are needed to come to a definite conclusion.

\section{Corresponding Information: Samaneh Salarvand, Department of Pathology, Imam Khomeini Hospital Complex, Tehran University of Medical Sciences, Tehran, Iran E-mail: $\underline{\text { Sm.salarvand@gmail.com }}$}

Copyright $(\odot$ 2022. This is an open-access article distributed under the terms of the Creative Commons Attribution- 4.0 International License which permits Share, copy and redistribution of the material in any medium or format or adapt, remix, transform, and build upon the material for any purpose, even commercially.

\section{Introduction}

It was on March 11, 2020, that the World Health Organization admitted COVID-19 is a pandemic (1). Over the succeeding months, the virus spread almost all countries around the world, though each country had a different pattern of COVID-19-related mortalities and morbidities. Contrary to what is expected based on the healthcare infrastructures of different countries, seemingly countries with lower indicators of socioeconomic development were less severely affected. They had a lower number of reported cases of COVID-19 per million population in comparison to the developed countries (2). Although such variations could be ascribed to the disparity between the availability of diagnostic techniques, financial resources, and access to healthcare services which lead to the underestimation of the actual prevalence, diversity of human pathogens in different geographic regions and concomitant immunological response may also play a protective role (3). One possible explanation for such a difference could be the number of people in a population who have disease-specific antibodies other than the coronavirus antibody, whether acquired through vaccination or previous infections. Viral hepatitis diseases caused by hepatitis A virus (HAV) and hepatitis E virus (HEV), two single-stranded RNA viruses spread by the fecal-oral route, are endemic in countries with low socioeconomic indices $(4,5)$. The extent of endemicity demonstrated by seroprevalence studies showed a direct correlation of the hygiene and the country's level of development with the prevalence 
of infection (6). Iran is one of those countries with a relatively high seroprevalence of $\operatorname{HAV}$ and $\operatorname{HEV}(7,8)$. This prevalence offers the opportunity to investigate the association of anti-HAV and -HEV antibodies with the susceptibility to coronavirus infection.

\section{Material and Methods}

This cross-sectional study was conducted on 90 randomly selected patients referred to Imam Khomeini Hospital Complex, Tehran, Iran. Among the hospitalized patients admitted to the infectious diseases ward with the clinical symptoms of COVID-19, 45 cases were selected for the study after performing the Reverse Transcription Polymerase Chain Reaction (RT-PCR) test and confirmation of the infection. Individuals referred to the severe acute respiratory infection (SARI) screening facility of the center who did not meet the criteria of suspected cases of COVID19 and tested negative for coronavirus infection through RT-PCR were selected for the control group. Individuals with immunodeficiency disorders, endstage renal diseases, organ transplants, and unstable vital signs were excluded from the study. The patients' chest CT scans were used as a confirmatory diagnostic test. Relevant demographic and clinical data were extracted from the patients' files.

RT-PCR tests were carried out on naso- and oropharyngeal samples which were collected following the WHO guidelines. RT-PCR test was performed on the samples utilizing the Novel Coronavirus (2019-nCOV) Nucleic Acid Diagnostic Kit (PCR-Fluorescence Probing) provided by Sansure Biotech (S3102E) (Changsha, China) which were run on CFX96 Touch Real-Time PCR Detection System (Bio-Rad Laboratories, Inc.) according to the manufacturer's instructions. Appropriate positive and negative 2019nCOV-PCR controls were run simultaneously.

For qualitative determination of total antibodies to HAV (IgM, IgG) and HEV (IgM, IgG, IgA) in the patients' sera, enzyme-linked immunosorbent assay (ELISA) was employed using HEV Ab Version ULTRA and HAV Ab kits provided by Dia.Pro Diagnostic Bioprobes Srl (Sesto, Italy) in accordance with the manufacturer's instructions. Tests were performed on the blood samples, which were taken for biochemical analyses. Test results were interpreted as a ratio of the cut-off value and the optical density measurement at $450 \mathrm{~nm}$ (OD $450 \mathrm{~nm}$ ) using a microplate reader with a $450 \mathrm{~nm}$ filter of the sample $(\mathrm{Co} / \mathrm{S}) . \mathrm{Co} / \mathrm{S}$ values of less than $0.9,0.9-1.1$, and more than 1.1 were interpreted as negative, equivocal, and positive, respectively. The cut-off value was calculated as negative plus positive control test results divided by 3.

The data were analyzed using SPSS software version 26 (SPSS Inc. Chicago, Ill., USA). Data were assessed for normality using the Kolmogorov-Smirnov test. Continuous variables were compared using the independent-samples $t$-test or Mann-Whitney $\mathrm{U}$ test where appropriate and reported as the median and interquartile range. Categorical variables were reported as frequencies. P-values less than 0.05 were considered statistically significant.

This study was performed after obtaining ethical approval from the Ethics Committee of Tehran University of Medical Sciences, Tehran, Iran.

\section{Results}

In the present study, 90 patients, including 45 cases ( 23 men and 22 women) and 45 controls (23 men and 22 women), were enrolled.

From 90 participants, 82 individuals $(91.1 \%)$ had positive HAV Ab test results with a median of 13.3 $(\mathrm{IQR}=11.3-15.7)$ and 8 individuals $(8.9 \%)$ had negative HAV Ab test results with a median of 0.42 (IQR = 0.4-0.46)

Among cases, 43 individuals (95.5\%) had positive, and two individuals (4.5\%) had negative HAV Ab test results, respectively. Among the controls, 39 individuals $(86.6 \%)$ had positive and six individuals (13.4\%) had negative HAV Ab test results, respectively. The median of HAV Ab levels was 13.6 $(\mathrm{Co} / \mathrm{S})(\mathrm{IQR}=11.5-16.9)$ and $13.2(\mathrm{Co} / \mathrm{S})(\mathrm{IQR}=10.7-$ 14.7) in cases and controls, respectively. The statistical analysis showed no significant difference between the two groups in term of HAV Ab level $(P=0.1)$ (Table 1).

Among all test subjects, $39(43.3 \%)$ and $51(56.7 \%)$ individuals had positive and negative HEV Ab test results, respectively, with a median of 0.5 (interquartile range $=0.18-6.6$ )

Among cases, $22(48.9 \%)$ and 23 (51.1\%), and among controls, $17(37.8 \%)$ and 28 (62.2\%) individuals had positive and negative $\mathrm{HEV} \mathrm{Ab}$ test results, respectively. The median of $\mathrm{HEV} \mathrm{Ab}$ level was $6.7(\mathrm{Co} / \mathrm{S})(\mathrm{IQR}=5.3-7.1)$ and $7.1(\mathrm{Co} / \mathrm{S})(\mathrm{IQR}=6.3-$ $7.5)$ in cases and controls, respectively. The statistical analysis showed no significant difference between the two groups regarding the HAV Ab level $(P=0.41)$ (Table 1).

Table 1. Hepatitis A and E virus antibodies level in COVID-19 patients and uninfected controls.

\begin{tabular}{|c|c|c|c|}
\hline & $\begin{array}{c}\text { Coronavirus infected patients }(\mathbf{n}=\mathbf{4 5}) \\
\text { Median (IQR) }\end{array}$ & $\begin{array}{c}\text { Control group (n= 45) } \\
\text { Median (IQR) }\end{array}$ & P-value \\
\hline HAV Ab level* & $13.6(5.4)$ & $13.2(4)$ & 0.1 \\
\hline HEV Ab level* & $6.7(1.8)$ & $7.1(1.3)$ & 0.41 \\
\hline
\end{tabular}

* Interpreted as the ratio of the cut-off value and the OD450nm of the sample (Co/S) 


\section{Discussion}

This study was conducted to evaluate the effect of preceding HAV and HEV infections on COVID-19 susceptibility. There have been some speculations about the potential protective role of antibodies and immune responses to HAV infections in patients who encountered the novel coronavirus (3). The hypothesis arose when a lower prevalence of coronavirus infection was observed in regions with poor socioeconomic conditions as well as a higher rate of $\mathrm{HAV}$ seropositivity in those areas. Despite its seemingly plausible relationship, our study could not detect a statistically significant association between the probability of infection with coronavirus and the level of serum hepatitis A and E antibodies.

Several pieces of evidence suggest the idea of probable protection against coronavirus infection in the presence of antibodies against viral hepatitis viruses (3, 9). In a review article, Sarialioglu et al. illustrated their unpublished observations regarding the low prevalence of coronavirus infection among patients who were under humoral or peritoneal dialysis (around 1 per 1000) compared to normal individuals. They concluded that this could be explained by the high HAV seroprevalence among these patients and pointed to the protective role of HAV antibodies and immune system stimulation following an HAV infection (3).

The rate of HAV and HEV infections are strongly correlated to the level of access to safe drinking water, socioeconomic status, and hygiene in the region. India, South African countries, and some countries in the Middle East have a high degree of endemicity and seropositivity for HAV and HEV infections. They suffer from poor public health conditions as well (6, 10). Interestingly, when the world encountered the coronavirus pandemic, these countries experienced a slighter increase in COVID-19 infections and a lower mortality rate compared to the developed countries(2, 11). This observation could be explained by a history of previous infections to other viruses which are endemic in those regions and the presence of crossprotective immunity as well as immunization schedules for viral infections. HAV and HEV infections are endemic in the above-mentioned countries, provoking both adaptive humoral and cellular immune responses(12). Furthermore, HAV and HEV vaccines are routinely administered in developing countries, which lead to outstanding immunogenicity, inducing a long-term sustained level of serum antibodies $(13,14)$.
The COVID-19 course seems to be milder in children, and this age group experiences a lower prevalence of coronavirus infection (15). The presence of antibodies against other viruses that are prevalent in this age group in developing countries could be considered as a reason. Although vaccination for HAV and $\mathrm{HEV}$ viruses is not universal in resource-poor countries, it is widely administered for children who are about one year old, leading to the advantageous immunogenicity that induces a high level of immune responses (16-18). Moreover, in developing countries, the vast majority of individuals are exposed to the hepatitis A virus in their childhood, so individuals in this age group have higher anti-hepatitis A virus antibody levels compared to older individuals (19). Therefore, one of the explanations for lower incidence and mortality of COVID-19 in children and young adults could be high levels of circulating antibodies for hepatitis A in these age groups.

The present study was designed to evaluate the potential relationship between the vulnerability to the novel coronavirus infection and the serum levels of hepatitis A and E antibodies. The results of this study that was carried out in one of the countries with a relatively high prevalence of $\mathrm{HAV}$ and $\mathrm{HEV}$ infections failed to show a statistically significant connection between COVID-19 susceptibility and HAV or HEV antibody levels.

We believe further studies with larger sample sizes in other geographic regions with higher endemicity of $\mathrm{HAV}$ and HEV infections are required before coming to a conclusion.

\section{Conclusion}

We believe further studies with larger sample sizes in other geographic regions with higher endemicity of $\mathrm{HAV}$ and HEV infections are required before coming to a conclusion.

\section{Acknowledgments}

The study was approved by the Ethical Committee of Tehran University of Medical Sciences, Tehran, Iran. The authors would like to extend their gratitude to the personnel of the laboratory of Imam Khomeini Hospital Complex for their help.

\section{Conflict of Interest}

The authors have no conflicts of interest to declare for this study.

\section{References}

1. WHO Director-General's opening remarks at the media briefing on COVID-19-11 March 2020: World Health Organization; 2020 [Available from: https://www.who.int/dg/speeches/detail/who- director-general-s-opening-remarks-at-themedia-briefingon-covid-19-11-march-2020 
2. COVID-19 CORONAVIRUS PANDEMIC [updated September 26, 2020. Available from: https://www.worldometers.info/coronavirus

3. Sarialioglu F, Apak FBB, Haberal M. Can Hepatitis A Vaccine Provide Protection Against COVID-19? Exp Clin Transplant. 2020;2:1413. [DOI:10.6002/ect.2020.0109] [PMID]

4. Wu D, Guo CY. Epidemiology and Prevention of Hepatitis A in Travelers. J Travel Med. 2013;20(6):394-9. [DOI:10.1111/jtm.12058] [PMID]

5. Tomar BS. Hepatitis E in India. Zhonghua Minguo xiao er ke yi xue hui za zhi [Journal] Zhonghua Minguo xiao er ke yi xue hui. 1998; 39(3):150-6.

6. World Health Organization. The global prevalence of hepatitis A virus infection and susceptibility: a systematic review [Internet]. World Health Organization. 2010.

7. Hoseini SG, Kelishadi R, Ataei B, Yaran M, Motlagh ME, Ardalan G, et al. Seroprevalence of hepatitis A in Iranian adolescents: is it time to introduce a vaccine? Epidemiol Infect. 2016; 144(2):291-6. [DOI:10.1017/S0950268815001302] [PMID]

8. Behzadifar M, Lankarani KB, Abdi S, Taheri Mirghaed M, Beyranvand G, Keshavarzi A, et al. Seroprevalence of Hepatitis E Virus in Iran: A Systematic Review and Meta-analysis. Middle East J Dig Dis. 2016;8(3):189-200. [DOI:10.15171/mejdd.2016.31] [PMID] [PMCID]

9. Sarialioğlu F, Belen FB, Hayran KM. Hepatitis A susceptibility parallels high COVID-19 mortality. Turk J Med Sci. 2021;51(1):382-4. [DOI:10.3906/sag-2007-133] [PMID] [PMCID]

10. Lee GY, Poovorawan K, Intharasongkroh D, Sa-Nguanmoo P, Vongpunsawad S, Chirathaworn C, et al. Hepatitis E virus infection: Epidemiology and treatment implications. World J Virol. 2015;4(4):343-55. [DOI:10.5501/wjv.v4.i4.343] [PMID] [PMCID]

11. Yaqinuddin A. Cross-immunity between respiratory coronaviruses may limit COVID-19 fatalities. Med Hypotheses. 2020;144:110049-.
[DOI:10.1016/j.mehy.2020.110049] [PMCID]

12. Walker CM. Adaptive Immune Responses in Hepatitis A Virus and Hepatitis E Virus Infections. Cold Spring Harb Perspect Med. 2019;9(9):a033472. [PMID] [PMCID] [DOI:10.1101/cshperspect.a033472]

13. Zhang J, Zhang X-F, Huang S-J, Wu T, Hu Y-M, Wang Z-Z, et al. Long-Term Efficacy of a Hepatitis E Vaccine. N Engl J Med. 2015;372 (10):914-22. [DOI:10.1056/NEJMoa1406011] [PMID]

14. Schmidtke P, Habermehl P, Knuf M, Meyer CU, Sänger R, Zepp F. Cell mediated and antibody immune response to inactivated hepatitis A vaccine. Vaccine. 2005;23(44):5127-32. [DOI:10.1016/j.vaccine.2005.06.022] [PMID]

15. Ludvigsson JF. Systematic review of COVID19 in children shows milder cases and a better prognosis than adults. Acta Paediatr. 2020;109(6):1088-95.

[DOI:10.1111/apa.15270] [PMID] [PMCID]

16. Arankalle V, Mitra M, Bhave S, Ghosh A, Balasubramanian S, Chatterjee S, et al. Changing epidemiology of hepatitis A virus in Indian children. Vaccine: Development and Therapy. 2014;4:7. [DOI:10.2147/VDT.S53324]

17. Aggarwal R, Goel A. Hepatitis A: epidemiology in resource-poor countries. Curr Opin Infect Dis. 2015;28(5):488-96. [PMID] [DOI:10.1097/QCO.0000000000000188]

18. Bell BP, Negus S, Fiore AE, Plotnik J, Dhotre $\mathrm{KB}$, Williams $\mathrm{J}$, et al. Immunogenicity of an inactivated hepatitis A vaccine in infants and young children. Pediatr Infect Dis J. 2007;26(2):116-22. [PMID] [DOI:10.1097/01.inf.0000253253.85640.cc]

19. ACHARYA SK, BATRA Y, BHATKAL B, OJHA B, KAUR K, HAZARI $S$, et al. Seroepidemiology of hepatitis A virus infection among school children in Delhi and north Indian patients with chronic liver disease: implications for HAV vaccination. J Gastroenterol Hepatol. 2003;18(7):822-7. [DOI:10.1046/j.14401746.2003.03051.x] [PMID] 